\title{
Lumbopelvic Stability and Trunk Muscle Contractility of Individuals with Chronic Ankle Instability
}

\author{
Ryan S McCann ${ }^{1}$, Kelly Johnson ${ }^{1}$, Ashley M B Suttmiller ${ }^{1}$ \\ 1 School of Rehabilitation Sciences, Old Dominion University \\ Keywords: transversus abdominis, self-reported function, lumbopelvic stability, diagnostic ultrasound, core endurance, ankle sprain \\ https://doi.org/10.26603/001c.22132
}

\section{International Journal of Sports Physical Therapy}

Vol. 16, Issue 3, 2021

\begin{abstract}
Background
Chronic ankle instability (CAI) results in hip neuromuscular impairments that can perpetuate dysfunction through reduced lumbopelvic stability and subsequent malpositioning of the lower body during functional movement. Lumbopelvic stability might be further impaired through changes in trunk muscular contractility. However, lumbopelvic stability and trunk muscle morphology have not been compared between individuals with and without CAI.
\end{abstract}

\section{Purpose}

To compare lumbopelvic stability and trunk muscle contractility between individuals with and without chronic ankle instability (CAI) and determine if lumbopelvic stability and trunk muscle contractility are associated with self-reported function.

\section{Study Design}

Case-control study.

\section{Methods}

Ten individuals with CAI, 10 ankle sprain copers (COP), and 10 healthy controls (CON) participated. Diagnostic ultrasound imaging was used to assess transversus abdominis (TrA) and lumbar multifidus (LM) muscle contractility. A percent change in contraction thickness from rested to contracted conditions was calculated for each muscle.

Lumbopelvic stability was assessed using unilateral hip bridge, trunk flexion endurance, Biering-Sorensen, and side plank tests. Self-reported function was measured with the Foot and Ankle Ability Measure Activity of Daily Living (FAAM-ADL) and Sport (FAAM-S) subscales. One-way ANOVAs and Cohen's d effect sizes compared scores on clinician and patient-reported outcomes between groups. Pearson product moment correlations analyzed associations between self-reported function and trunk muscle contractility and lumbopelvic stability. Significance was set a priori at $\mathrm{P}<0.05$

\section{Results}

COP had significantly greater TrA contractility than CAI $(\mathrm{P}<0.01, d=2.65[1.45,3.85])$ and $\operatorname{CON}(\mathrm{P}=0.03, d=1.05[0.08,1.94])$. Although not statistically significant, a large effect size suggest that CAI had lower TrA contractility than CON ( $\mathrm{P}=0.12, d=0.92[-0.03,1.80])$. No differences existed for LM contractility or lumbopelvic stability tests. A moderate direct

\footnotetext{
Corresponding Author: 
correlation $(\mathrm{r}=0.65, \mathrm{P}=0.04)$ existed between CON's TrA contractility and FAAM-ADL scores.

\section{Conclusion}

Deficits in TrA contractility are a novel finding among individuals with CAI. While LM contractility and lumbopelvic stability did not differ between groups, future research should continue to examine their relevance to CAI.

\section{Level of Evidence}

\section{INTRODUCTION}

Chronic ankle instability (CAI) is a significant musculoskeletal condition that affects up to $70 \%$ of the estimated 23,000 individuals that sustain an ankle sprain in the United States daily. ${ }^{1,2}$ CAI is associated with various impairments that promote frequent ankle sprain recurrences, episodes of "giving way," and feelings of instability. ${ }^{3,4}$ In addition to being highly common, CAI is associated with decreased levels of physical activity and quality of life throughout the lifespan and increased risk for joint osteoarthritis. ${ }^{3}$ Conversely, a portion of individuals with a history of ankle sprain, deemed copers, respond more favorably by avoiding these long-term consequences. ${ }^{5}$

Copers and individuals with CAI are distinguishable through surveys of self-reported function (i.e. ability to complete activities of daily living and sport $)^{6}$ as well as tests of neuromuscular control. ${ }^{3,7}$ Such comparisons are valuable to clinicians designing and evaluating therapeutic interventions intended to eliminate characteristics of CAI and foster those of copers. Most of the comparisons between individuals with CAI and copers have examined residual impairments in the ankle joint, and thus, most rehabilitation protocols focus exclusively on restoring function of the ankle. ${ }^{8}$ Increasingly, individuals with CAI are reported to have hip muscular impairments, ${ }^{9,10}$ which can perpetuate CAI through reduced stability of the trunk and pelvis (lumbopelvic stability) and resultant malpositioning of the lower body. 11,12

Although CAI is potentially exacerbated by reduced lumbopelvic stability, this association remains predominantly theoretical. Lumbopelvic stability can be evaluated through tests that challenge an individual's ability to maintain trunk and hip alignment, but to date, no studies have compared performance between individuals with and without CAI. Furthermore, while lumbopelvic stability is likely affected by hip muscular impairments, it is also highly influenced by the trunk musculature. However, minimal research exists regarding trunk muscle morphology in individuals with CAI. Previous studies have reported that individuals with CAI have greater rates of low back pain, ${ }^{13}$ delays in trunk muscle activation, ${ }^{14}$ and reduced contractility of the diaphragm muscle. ${ }^{15}$ Trunk muscle contractility is important for lumbopelvic stability, but contractility of essential stabilizers, such as the transversus abdominis and lumbar multifidus, has not been examined in individuals with CAI.

Therefore, the purpose of this study was to compare lumbopelvic stability and trunk muscle contractility between individuals with and without CAI. The authors hypothesized that individuals with CAI would have reduced lum- bopelvic stability and trunk muscle contractility compared to copers and healthy controls. Identification of deficits in lumbopelvic stability and trunk muscle contractility would direct clinicians to new rehabilitation strategies that could contribute to comprehensive care for individuals with CAI. Additionally, the authors intend to determine if deficits in lumbopelvic stability and trunk muscle contractility were associated with reduced self-reported function. The authors hypothesized that greater lumbopelvic stability and trunk muscle contractility would be associated with better self-reported function in individuals with CAI, copers, and healthy controls. With this analysis, the authors will elucidate how new interventions that target the lumbopelvic region might influence patients' perceived abilities to engage in activities of daily living and sport.

\section{METHODS}

\section{PARTICIPANTS}

Using a case-control study design, 30 participants were separated into CAI, coper (COP), and control (CON) groups, using criteria established by the International Ankle Consortium. ${ }^{4,5}$ Inclusionary criteria for all groups consisted of being between ages 18 and 40 and completing at least 30 minutes of physical activity three times per week. Exclusion criteria consisted of a history of balance or vestibular disorders, previous spine or lower extremity fracture or surgery, low back pain in the previous six months, concussion in the previous 6 months, and spine and lower extremity musculoskeletal and neurovascular disorders (besides ankle sprain) in the previous two years. All participants read and signed an informed consent document approved by the university's Institutional Review Board prior to beginning any study procedures.

\section{PROCEDURES}

Each participant reported to the Athletic Training Education Laboratory for a single session and completed the study procedure in the following order: 1) self-reported function, 2) trunk muscle contractility, 3) lumbopelvic stability. The two muscle contractility tests and four lumbopelvic stability tests (unilateral hip bridge endurance, trunk flexion endurance, Biering-Sorensen, and side plank) were completed in an order determined by a random number generator. One trial of each lumbopelvic stability test was completed with 1-minute rest intervals between trials. Tests were conducted on the involved side for CAI and COP groups, and a randomly selected side for the CON group. In the case a member of the CAI group had bilateral CAI, the side with 
the most episode of giving way was tested. Participants reported their level of ankle-specific function with the Foot and Ankle Ability Measure (FAAM) questionnaire. ${ }^{6}$ Participants completed both Activity of Daily Living (FAAM-ADL) and Sport (FAAM-S) subscales. Calculated scores for each subscale ranged from 0 (complete loss of function) to 100 (no loss of function).

Diagnostic ultrasound imaging was used to analyze contractility of the transversus abdominis (TrA) and lumbar multifidus (LM) muscles in the trunk. A portable ultrasound unit (LOGIQ e 2008; GE Healthcare, Wauwatosa, WI) and a linear-array transducer (12L-RS, GE Healthcare, Wauwatosa, WI) visualized and recorded images of each muscle. Thickness of both muscles were measured using images taken at rest and while contracted using procedures described previously. ${ }^{16}$ An average of three measurements for each muscle in rested and contracted conditions was calculated. A percent change in contraction thickness from rested to contracted conditions was calculated for each muscle using the following formula:

$\left(\right.$ mean $_{\text {contacted }}-$ mean $\left._{\text {rested }}\right) /$ mean $_{\text {rested }}$ x $100 .{ }^{16}$

The unilateral hip bridge endurance test was performed on the floor with the participant supine and arms across their chest. The participant performed a double-leg bridge maneuver until a neutral spine and pelvis were achieved. To maintain a neutral spine, the examiner aligned a target with the anterior superior iliac spine of the participant's nontest limb. Once a neutral position was established, the participant extended the knee of the non-test limb so that their thighs remained parallel. While their hips were supported by the test limb, the participant held this position for maximum time.

The trunk flexion endurance test required the participant to sit against a plank with the trunk in $60^{\circ}$ of flexion relative to the floor. ${ }^{17}$ The participant's knees and hips were flexed to $90^{\circ}$ and hands were folded across their chest. The examiner removed the plank away from the participant's back, and the participant was instructed to maintain $60^{\circ}$ of flexion for maximum time.

The Biering-Sorensen test required the participant to lay prone on a treatment table with their trunk unsupported. The participant was secured with straps across their waist and lower legs. ${ }^{18}$ The participant folded their arms across their chest and maintained their unsupported trunk in a neutral position for maximum time.

The side plank test required participants to assume a side-lying position with their involved side down. ${ }^{18}$ The participant raised their hips and trunk off the table, supporting their body in a neutral position through their feet and elbow, and maintained that position for maximum time.

\section{STATISTICAL ANALYSIS}

Shapiro-Wilk tests were used to examine normality of demographics and primary outcomes of each group. Since data were found to be normally distributed $(\mathrm{P}>0.05)$, separate one-way ANOVAs were used to compare demographics, self-reported function, trunk muscle contractility, and lumbopelvic stability between groups. For significant main effects, Tukey post-hoc tests examined pairwise compar- isons. Cohen's $d$ effect sizes (small $=0.2-0.49$, moderate $=0.5-0.79$, large $>0.8$ ) and 95\% confidence intervals examined the magnitude of significant pairwise differences. Pearson product moment correlations (negligible $<0.3$, low $=0.3-0.49$, moderate $=0.5-0.69$, high $=0.7-0.89$, very high $=$ 0.9-1.0) analyzed associations between self-reported function and trunk muscle contractility and lumbopelvic stability. Significance was set $a$ priori at $\mathrm{P}<0.05$. All statistical analyses were conducted using IBM SPSS Statistics, version 24 (IBM Corporation, Armonk, NY).

\section{RESULTS}

Between groups comparisons of demographics and primary outcomes are presented in Table 1. No significant differences in demographics were present. Significant main effects were present for TrA contractility, unilateral hip bridge, and scores on both FAAM subscales. Pairwise comparisons revealed that COP had significantly greater TrA contractility than CAI ( $\mathrm{p}<0.01, d=2.65[1.45,3.85])$ and CON ( $\mathrm{p}=0.03, d=1.05[0.08,1.94])$. No statistically significant difference in TrA contractility was present between CAI and CON ( $p=0.12, d=0.92[-0.03,1.80])$. No significant pairwise comparisons were present for the unilateral hip bridge test. CAI had significantly lower FAAM-ADL scores than COP ( $\mathrm{p}=0.02, d=-1.07[-1.96,-0.10])$ and CON ( $\mathrm{p}=$ $0.01, d=-1.15[-2.05,-0.16])$ and significantly lower FAAM-S scores than COP $(\mathrm{p}=0.04, d=-0.94[-1.82,0.02])$ and CON (p $<0.01, d=-1.45[-2.36,-0.41])$.(Table 2) For CON, a moderate direct correlation existed between TrA contractility with FAAM-ADL scores (Table 3). No other significant correlations were present.

\section{DISCUSSION}

The primary finding of this study is that COP exhibited significantly greater TrA contractility compared to CAI and CON. The reason for COP's superior TrA contractility is beyond the intent of this study, but potential contributors include elevated pre-injury TrA contractility, inherent resistance to post-injury neural inhibition and central nervous system reorganization, and completion of thorough, highquality rehabilitation. Regardless of the reason, designation as a coper is considered the antithesis to developing CAI, ${ }^{19}$ and thus, copers' neuromuscular alterations are regarded as positive adaptations that protect against persistent consequences of the initial ankle sprain. Numerous studies have reported that copers exhibit superior sensorimotor control compared to individuals with CAI and healthy controls. ${ }^{5}$ The identification of enhanced TrA contractility in copers suggests that targeting it during ankle sprain rehabilitation might contribute to avoidance of CAI, however, this remains unconfirmed.

While there was not a significant difference between CAI and CON, large effect sizes suggest that CAI might have had clinically meaningful reductions in TrA contractility compared to COP and CON. The TrA primarily acts as a spinal stabilizer by increasing intra-abdominal pressure and is an important part of feedforward motor control when anticipating an external perturbation or preparing for move- 
Table 1: Between-group comparisons of demographics

\begin{tabular}{|c|c|c|c|c|c|}
\hline & $\mathrm{CAI}(\mathrm{n}=10)$ & $\operatorname{COP}(n=10)$ & $\begin{array}{c}\mathrm{CON} \\
(n=10)\end{array}$ & $\begin{array}{l}\text { ANOVA } \\
\quad(F)\end{array}$ & $\begin{array}{c}\mathrm{P}- \\
\text { value }\end{array}$ \\
\hline Age (yrs.) & $23.9 \pm 4.4$ & $23.0 \pm 3.0$ & $23.2 \pm 3.3$ & 0.20 & 0.82 \\
\hline Height (cm) & $168.6 \pm 9.8$ & $173.0 \pm 6.5$ & $166.6 \pm 6.1$ & 1.82 & 0.18 \\
\hline Mass (kg) & $81.5 \pm 19.1$ & $76.0 \pm 13.4$ & $68.7 \pm 16.3$ & 1.54 & 0.23 \\
\hline $\operatorname{Sex}(M / F)$ & $5 \mathrm{M} / 5 \mathrm{~F}$ & $5 \mathrm{M} / 5 \mathrm{~F}$ & $3 \mathrm{M} / 7 \mathrm{~F}$ & & \\
\hline Previous Ankle Sprains (\#) & $2.9 \pm 1.8^{*}$ & $1.7 \pm 0.7^{*}$ & $0.0 \pm 0.0$ & 17.37 & $<0.01$ \\
\hline $\begin{array}{l}\text { Time Since Most Recent Ankle Sprain } \\
\text { (months) }\end{array}$ & $\begin{array}{l}41.3 \\
\pm 33.3^{*}\end{array}$ & $\begin{array}{l}62.4 \pm \\
35.1^{*}\end{array}$ & $0.0 \pm 0.0$ & 12.91 & $<0.01$ \\
\hline
\end{tabular}

*Statistically different from the CON group ( $<<0.05)$

Abbreviation: CAI, chronic ankle instability; COP, coper; CON, control

Table 2: Between-group comparisons of primary outcomes

\begin{tabular}{lccccc}
\hline & CAI $(\mathrm{n}=10)$ & COP $(\mathrm{n}=10)$ & CON $(\mathrm{n}=10)$ & ANOVA (F) & P-value \\
\hline LM (\% change) & $13.5 \pm 9.2$ & $14.9 \pm 7.5$ & $16.4 \pm 10.9$ & 0.26 & 0.77 \\
TrA (\% change) & $32.6 \pm 17.9 \dagger$ & $114.0 \pm 37.6^{*}$ & $67.4 \pm 50.2$ & 11.73 & $<0.01$ \\
Unilateral Hip Bridge Test (s) & $29.5 \pm 21.3$ & $54.6 \pm 33.4$ & $28.6 \pm 13.8$ & 3.71 & 0.04 \\
Beiring-Sorensen Test (s) & $101.5 \pm 44.5$ & $144.0 \pm 77.6$ & $139.2 \pm 62.6$ & 1.37 & 0.27 \\
Side Plank Test (s) & $68.4 \pm 67.2$ & $84.6 \pm 36.6$ & $62.7 \pm 29.6$ & 0.58 & 0.57 \\
Trunk Flexion Endurance Test (s) & $219.4 \pm 103.2$ & $188.5 \pm 91.4$ & $178.3 \pm 91.5$ & 0.50 & 0.61 \\
FAAM-ADL (\%) & $89.4 \pm 11.9^{*} \dagger$ & $98.6 \pm 2.3$ & $99.2 \pm 1.6$ & 6.00 & $<0.01$ \\
FAAM-S (\%) & $81.8 \pm 16.2^{*} \dagger$ & $94.3 \pm 9.5$ & $98.6 \pm 2.5$ & 6.34 & $<0.01$ \\
\hline
\end{tabular}

*Statistically different from the CON group $(\mathrm{p}<0.05)$

†Statistically different from the COP group $(\mathrm{p}<0.05)$

Abbreviation: CAI, chronic ankle instability; COP, coper; CON, control; LM, lumbar multifidus; TrA, transversus abdominis; FAAM-ADL, Foot and Ankle Ability Measure Activity of Daily Living subscale; FAAM-S, Foot and Ankle Ability Measure Sport subscale

ment. ${ }^{20}$ The ability of the TrA to increase intra-abdominal pressure is contingent upon the diaphragm's ability to contract and maintain the position of the abdominal contents. Terada et al. ${ }^{15}$ previously reported that individuals with CAI had reduced left hemidiaphragm contractility, which might further impair trunk stability when combined with reduced TrA contractility. Gong ${ }^{21}$ provided evidence of the TrA's contribution to postural control performance in a study that reported a low, significant correlation between TrA thickness and static balance performance in healthy females. Another study ${ }^{22}$ reported that increasing postural demand during static stance resulted in increased TrA activation of healthy males. Collectively, these studies suggest that balance impairments, which are commonly identified among individuals with CAI, might be partially attributable to dysfunction of the TrA. However, since postural control was not evaluated in the current study, the link between TrA contractility and balance deficits of individuals with CAI could not be determined by this study. While contractile impairments of the TrA appear to exist among individuals with CAI, the etiology of this alteration cannot be determined through the current retrospective study design. Evidence exists for neuromuscular impairments being precursors to and results of CAI, ${ }^{23,24}$ so more work is needed to identify the course of TrA contractility deficits.
Converse to the TrA, no differences in LM contractility were present between groups. While the LM also has a part in feedforward activation when preparing for lower extremity movement, its contraction does not occur as early as the TrA, potentially indicating a lesser role in initiating lumbopelvic stability. ${ }^{25}$ Despite its later activation, the role of the LM in spinal stabilization is well documented; of note, reduced cross sectional area of LM is implicated in low back pain. ${ }^{26}$ Limited evidence exists linking LM contractility, low back pain, and CAI. Nadler et al. ${ }^{13}$ reported that collegiate athletes with lower extremity injuries, including CAI, required more treatment for low back pain compared to those without lower extremity injuries. While it is conceivable that dysfunction of the LM could be more prominent among individuals with CAI, these data do not support this association. A potential reason for the lack of differences in LM contractility is the exclusionary criteria, which eliminated individuals with low back pain. Future studies should examine alterations in LM contractility among multiple groups with concurrent and asynchronous presentations of CAI and low back pain.

In addition to LM contractility, none of the lumbopelvic stability tests differed between groups. Lumbopelvic stability is widely considered a requisite to neuromuscular control of the lower extremity by providing a stable foundation 
Table 3: Pearson Product Moment Correlations between FAAM scores and clinical outcomes.

\begin{tabular}{|c|c|c|c|c|c|}
\hline & & \multicolumn{2}{|c|}{ FAAM-ADL (\%) } & \multicolumn{2}{|c|}{ FAAM-S (\%) } \\
\hline & & $r$ & $P$ & $r$ & $P$ \\
\hline \multirow{3}{*}{ LM (\% change) } & CAI & -0.16 & 0.66 & -0.25 & 0.49 \\
\hline & COP & 0.07 & 0.84 & -0.07 & 0.86 \\
\hline & CON & 0.19 & 0.60 & 0.30 & 0.40 \\
\hline \multirow{3}{*}{ TrA (\% change) } & CAI & 0.28 & 0.44 & 0.33 & 0.35 \\
\hline & $C O P$ & -0.55 & 0.10 & -0.49 & 0.15 \\
\hline & CON & 0.65 & $0.04^{*}$ & 0.52 & 0.12 \\
\hline \multirow{3}{*}{ Unilateral Hip Bridge (s) } & CAI & 0.50 & 0.14 & 0.35 & 0.33 \\
\hline & COP & 0.08 & 0.82 & 0.16 & 0.66 \\
\hline & CON & 0.14 & 0.71 & -0.44 & 0.20 \\
\hline \multirow{3}{*}{ Beiring-Sorensen (s) } & CAI & 0.44 & 0.20 & 0.30 & 0.39 \\
\hline & COP & 0.35 & 0.33 & 0.37 & 0.29 \\
\hline & CON & 0.50 & 0.14 & 0.10 & 0.79 \\
\hline \multirow{3}{*}{ Side Plank (s) } & CAI & 0.48 & 0.15 & 0.47 & 0.17 \\
\hline & COP & -0.18 & 0.62 & -0.11 & 0.754 \\
\hline & CON & 0.30 & 0.41 & -0.21 & 0.56 \\
\hline \multirow{3}{*}{ Trunk Flexion Endurance (s) } & CAI & 0.01 & 0.98 & -0.30 & 0.40 \\
\hline & COP & 0.10 & 0.79 & 0.14 & 0.71 \\
\hline & CON & 0.16 & 0.66 & -0.50 & 0.14 \\
\hline
\end{tabular}

*Statistically significant correlation $(\mathrm{p}<0.05)$

Abbreviation: CAI, chronic ankle instability; COP, coper; CON, control; LM, lumbar multifidus; TrA, transversus abdominis; FAAM-ADL, Foot and Ankle Ability Measure Activity of Daily Living subscale; FAAM-S, Foot and Ankle Ability Measure Sport subscale

upon which to generate motion and transmit force. Increased gluteal muscle activation latencies 9,27 and decreased hip strength ${ }^{10}$ previously reported in individuals with CAI signify that deficits commonly exist in stabilizers of the lumbopelvic complex. Thus, the authors hypothesized that among individuals with CAI, reduced lumbopelvic stability would be present and potentially contribute to reduced neuromuscular control of the lower extremity. Others have attempted to, but were unsuccessful in determining if reduced baseline scores on trunk flexion endurance, Biering-Sorensen, and side plank tests increase risk of lower extremity injuries. ${ }^{28}$ The trunk flexion endurance test had some mixed findings, but the lumbopelvic stability tests did not demonstrated predictive value for injury. Although the ability of these tests to predict ankle sprains specifically has not been explored, the findings of the current and previous studies suggest that lumbopelvic stability might have limited relevance to CAI. However, it is also possible that the static endurance tests were simply not representative of lumbopelvic stability deficits of individuals with CAI. A previous study ${ }^{14}$ of individuals with functional ankle instability demonstrated increased latency times of the erector spinae and rectus abdominis muscles during a trunk unloading task compared to healthy controls. Additionally, increased trunk extensor latency was associated with greater time required to stabilize following a jump landing. A similar laboratory test identified increased sagittal and frontal plane displacements of the trunk after sudden unloading as significant risk factors for knee in- juries in female collegiate athletes. ${ }^{29}$ The unanticipated nature of the unloading tasks in the previous studies provided unique challenges not present in the stability tests that incorporated no unanticipated perturbations. Unanticipated landing tasks have previously resulted in increased lower extremity muscle activation latency, and thus reduced preparation of the sensorimotor system, compared to anticipated landing tasks. ${ }^{30}$ If unanticipated perturbations are needed to detect lumbopelvic stability deficits of individuals with CAI, then it is unlikely that the selected tests would do so. Although the selected lumbopelvic stability tests failed to differentiate individuals with and without CAI, the authors recommend that the efficacy of other lumbopelvic stability measures should be explored. Tests that involve assessment of dynamic lumbopelvic stability will likely be more applicable to the functional demands of physically active individuals.

Only one statistically significant correlation was found, in which the CON group's TrA contractility was moderately associated with FAAM-ADL scores. This finding indicates that healthy individuals with greater TrA contractility had greater self-reported physical function during ADLs. Thus, in the CON group, the TrA likely serves as a rigid support to generate proximal stability and better contribute to neuromuscular control during functional tasks. While this association was expected, the authors anticipated lumbopelvic stability tests and LM contractility would have additional relationships with self-reported function. It is unclear why no other meaningful correlations were discovered, but as 
discussed earlier, the selected lumbopelvic stability tests might not have been truly representative of lumbopelvic stability of copers or individuals with CAI, and thus, demonstrate minimal influence over self-reported function. Additionally, it is possible that participants with a previous ankle sprain experienced central nervous system reorganization that limited the influence of the LM and TrA on selfreported function.

Limitations must be acknowledged in the present in this study. First, the retrospective study design limits the authors' ability to determine if TrA contractility deficits originated before or after the onset of CAI. While participants were required to maintain a minimum level of physical activity, further variations in exercise mode, intensity, frequency, and duration that were not accounted for might have influenced the study results. Additionally, variations in physical activity might have affected the participants' group placement; those that avoid certain physical activities might be less likely to sprain their ankle or experience other symptoms of CAI. The lack of examiner blinding could have influenced observations when conducting clinical tests. The authors attempted to evaluate lumbopelvic stability with multiple clinically applicable tests, but as explained earlier, the tests might not have sufficiently challenged the sensorimotor system of the participants enough to detect between-group differences.

\section{CONCLUSION}

Copers exhibited superior TrA contractility compared to individuals with CAI and healthy controls. This finding is potentially indicative of a positive neuromuscular adaptation in copers that provides protection against the onset of CAI. Conversely, individuals with CAI might be less able to develop this adaptation, which could contribute to the persistence of CAI. There is no distinct reason that the CAI population lacks this adaptation, but inherent resistance or insufficient rehabilitation are potential contributors. While TrA contractility was not influential to self-reported function of copers or individuals with CAI, further research should examine the value of enhancing this outcome during rehabilitation of patients with CAI. While no group differences in lumbopelvic stability were present, future studies should continue to explore the importance of lumbopelvic stability in CAI rehabilitation.

\section{CONFLICTS OF INTEREST}

The authors of this manuscript have no conflicts of interest to disclose.

Submitted: September 02, 2020 CDT, Accepted: November 26, 2020 CDT 


\section{REFERENCES}

1. Braun BL. Effects of ankle sprain in a general clinic population 6 to 18 months after medical evaluation. Arch Fam Med. 1999;8(2):143-148.

2. Hertel J. Functional Anatomy, Pathomechanics, and Pathophysiology of Lateral Ankle Instability. J Athl Train. 2002;37(4):364-375.

3. Gribble PA, Bleakley CM, Caulfield BM, et al. Evidence review for the 2016 International Ankle Consortium consensus statement on the prevalence, impact and long-term consequences of lateral ankle sprains. Br J Sports Med. 2016;50(24):1496-1505. doi:1 $\underline{0.1136 / \text { bjsports-2016-096189 }}$

4. Gribble PA, Delahunt E, Bleakley C, et al. Selection criteria for patients with chronic ankle instability in controlled research: a position statement of the International Ankle Consortium. J Orthop Sports Phys Ther. 2013;43(8):585-591. doi:10.2519/jospt.2013.030 $\underline{3}$

5. Wikstrom EA, Brown CN. Minimum reporting standards for copers in chronic ankle instability research. Sports Med. 2014;44(2):251-268. doi:10.100 7/s40279-013-0111-4

6. Carcia CR, Martin RL, Drouin JM. Validity of the Foot and Ankle Ability Measure in athletes with chronic ankle instability. J Athl Train.

2008;43(2):179-183. doi:10.4085/1062-6050-43.2.179

7. Plante JE, Wikstrom EA. Differences in clinicianoriented outcomes among controls, copers, and chronic ankle instability groups. Phys Ther Sport. 2013;14(4):221-226. doi:10.1016/i.ptsp.2012.09.005

8. Kosik KB, McCann RS, Terada M, Gribble PA. Therapeutic interventions for improving self-reported function in patients with chronic ankle instability: a systematic review. Br J Sports Med.

2017;51(2):105-112. doi:10.1136/bjsports-2016-09653 4

9. Beckman SM, Buchanan TS. Ankle inversion injury and hypermobility: effect on hip and ankle muscle electromyography onset latency. Arch Phys Med Rehabil. 1995;76(12):1138-1143.

10. McCann RS, Crossett ID, Terada M, Kosik KB, Bolding BA, Gribble PA. Hip strength and star excursion balance test deficits of patients with chronic ankle instability. J Sci Med Sport.

2017;20(11):992-996. doi:10.1016/j.jsams.2017.05.005
11. Fuller EA. Center of pressure and its theoretical relationship to foot pathology. J Am Podiatr Med Assoc. 1999;89(6):278-291. doi:10.7547/87507315-8 9-6-278

12. MacKinnon CD, Winter DA. Control of whole body balance in the frontal plane during human walking. $J$ Biomech. 1993;26(6):633-644. doi:10.1016/0021-929 0(93) $90027-\mathrm{C}$

13. Nadler SF, Wu KD, Galski T, Feinberg JH. Low back pain in college athletes. A prospective study correlating lower extremity overuse or acquired ligamentous laxity with low back pain. Spine. 1998;23(7):828-833.

14. Marshall PW, McKee AD, Murphy BA. Impaired trunk and ankle stability in subjects with functional ankle instability. Med Sci Sports Exerc.

2009;41(8):1549-1557. doi:10.1249/MSS.0b013e31819 $\underline{\mathrm{d} 82 \mathrm{e} 2}$

15. Terada M, Kosik KB, McCann RS, Gribble PA. Diaphragm contractility in individuals with chronic ankle instability. Med Sci Sports Exerc. 2016;48(10):2040-2045. doi:10.1249/MSS.0000000000 $\underline{000994}$

16. Koppenhaver SL, Hebert JJ, Fritz JM, Parent EC, Teyhen DS, Magel JS. Reliability of rehabilitative ultrasound imaging of the transversus abdominis and lumbar multifidus muscles. Arch Phys Med Rehabil. 2009;90(1):87-94. doi:10.1016/i.apmr.2008.06.022

17. McGill SM, Childs A, Liebenson C. Endurance times for low back stabilization exercises: clinical targets for testing and training from a normal database. Arch Phys Med Rehabil. 1999;80(8):941-944.

18. Palmer TG, Uhl TL. Interday reliability of peak muscular power outputs on an isotonic dynamometer and assessment of active trunk control using the chop and lift tests. J Athl Train. 2011;46(2):150-159. doi:1 $\underline{0.4085 / 1062-6050-46.2 .150}$

19. Hertel J, Corbett RO. An updated model of chronic ankle instability. J Athl Train. 2019;54(6):572-588. do i:10.4085/1062-6050-344-18

20. Hodges PW. Is there a role for transversus abdominis in lumbo-pelvic stability? Man Ther. 1999;4(2):74-86. doi:10.1054/math.1999.0169

21. Gong W. Correlations between transversus abdominis thickness, lumbar stability, and balance of female university students. J Phys Ther Sci. 2013;25(6):681-683. doi:10.1589/jpts.25.681 
22. Crommert ME, Ekblom MM, Thorstensson A. Activation of transversus abdominis varies with postural demand in standing. Gait Posture. 2011;33(3):473-477. doi:10.1016/i.gaitpost.2010.12.02 $\underline{8}$

23. Delahunt E, Remus A. Risk factors for lateral ankle sprains and chronic ankle instability. J Athl Train. 2019;54(6):611-616. doi:10.4085/1062-6050-44-18

24. Ward S, Pearce AJ, Pietrosimone B, Bennell K, Clark R, Bryant AL. Neuromuscular deficits following peripheral joint injury: A neurophysiological hypothesis. Muscle Nerve. 2015;51(3):327-332. doi:1 $\underline{0.1002 / \text { mus. } 24463}$

25. Hodges PW, Richardson CA. Contraction of the abdominal muscles associated with movement of the lower limb. Phys Ther. 1997;77(2):132-142. doi:10.109 3/ptj/77.2.132

26. Fortin M, Macedo LG. Multifidus and paraspinal muscle group cross-sectional areas of patients with low back pain and control patients: a systematic review with a focus on blinding. Phys Ther. 2013;93(7):873-888. doi:10.2522/ptj.20120457
27. Bullock-Saxton JE. Local sensation changes and altered hip muscle function following severe ankle sprain. Phys Ther. 1994;74(1):17-28.

28. De Blaiser C, Roosen P, Willems T, Danneels L, Bossche LV, De Ridder R. Is core stability a risk factor for lower extremity injuries in an athletic population? A systematic review. Phys Ther Sport. 2018;30:48-56. doi:10.1016/i.ptsp.2017.08.076

29. Zazulak BT, Hewett TE, Reeves NP, Goldberg B, Cholewicki J. Deficits in neuromuscular control of the trunk predict knee injury risk: a prospective biomechanical-epidemiologic study. Am J Sports Med. 2007;35(7):1123-1130. doi:10.1177/036354650730158 $\underline{5}$

30. Dicus JR, Seegmiller JG. Unanticipated ankle inversions are significantly different from anticipated ankle inversions during drop landings: overcoming anticipation bias. J Appl Biomech. 2012;28(2):148-155. doi:10.1123/jab.28.2.148 